\title{
Das (vermeintlich) ungestillte Verlangen nach Süßem?
}

\author{
Peter Brandt
}

Eingegangen: 25 January 2010/ Online publiziert: 11 February 2010

(C) Springer Basel AG 2010

Will man sich mit dem „Süßem“ eingehender beschäftigen, so assoziiert man sicher mit „Süßem“ zugleich Wohlgenuss wie Überdruss, ganz zwangsläufig illuminiert in rosa- bis pink-farbenen Schattierungen. Dieses ambivalente Verhalten gegenüber dem „Süßen“ ist aber erst ein Phänomen der letzen 50 Jahre in Deutschland (und wohl auch in großen Teilen der EU). Davor galt „Zuckerwerk“ als etwas Besonderes, mit dem insbesondere die Kinder zu Weihnachten erfreut wurden (wie z. B. bei Storm, Mörike oder Raabe nachzulesen ist) oder das zuvor als Luxusartikel im höfischen Bereich zur Erquickung der Hofdamen etc. herhalten musste.

Diese positiv belastete Werte-Assoziation des Süßen hat sich auch in die heutige Zeit hinübergerettet, wenn auch in stark geminderter Form. Wenn z. B. Kindergruppen zum Halloween lautstark fordern „Gib uns Süßes, sonst bekommst Du Saures“, so ist diese Drohung in den meisten Fällen sicher nur ein Ritual, dessen sich die Kindergruppen nur vage bewusst sein werden, aber dennoch werden mit dieser Ankündigung die positiven Aspekte des „Süßen“ - gespiegelt an den negativen Aspekten des „Sauren“ - überaus prägnant betont. In ähnlicher Weise - wenn auch nur im übertragenen Sinn gemeint - wird auch gelegentlich das Begriffspaar „Zuckerbrot und Peitsche“ verwendet.

Prof. Dr. Dr. P. Brandt ( $\square)$

BVL, Redakteur des JVL und der BVL-Reporte,

Wissenschaftliche Redaktionsgruppe,

Forschungskoordination,

Mauerstraße 39-42, 10117 Berlin, Germany

e-mail: Peter.Brandt@bvl.bund.de
Als kleiner Exkurs sei es an dieser Stelle erlaubt darauf hinzuweisen, dass die scheinbaren negativen Aspekte des „Sauren“ so unabdingbar nicht sind, denkt man z. B. an den spontan erwachenden Heißhunger (z. B. von Schwangeren) auf den Verzehr saurer Speisen und damit keineswegs eine negative Einstufung des „Sauren“. Insofern kann die Bedeutung des „Süßen“ für die menschliche Sensorik und dem vermeintlich ungestilltem Verlangen nach ihm keineswegs als unvermeidbar gelten.

Zieht man daraufhin die weltweiten Produktionsraten für Zucker zu Rate, wie sie von der Wirtschaftlichen Vereinigung Zucker für den Zeitraum seit Ende der achtziger Jahre im Internet ${ }^{1}$ publiziert werden, so zeigt sich, dass die weltweite Zuckererzeugung ansteigt (Abb. 1), was vorwiegend auf die Zunahme der Rohrzuckererzeugung zurückzuführen ist. Im Gegensatz dazu bewegt sich die Rübenzuckererzeugung im demselben Zeitraum mit rund 32 Mill. $\mathrm{t}$ auf nahezu unverändertem Niveau. Für 2008/2009 wird eine Erzeugung von Rohrzucker in Höhe von 124,6 Mill. t Rohwert und von Rübenzucker in Höhe von 31,7 Mill. t Rohwert erwartet. Der Anteil des Rübenzuckers an der Gesamterzeugung wird auf $20 \%$ geschätzt.

Für unser Thema sind aber die Angaben zum Zuckerverbrauch pro Kopf in den letzten fünf Jahren von noch größerem Interesse (Abb. 2). Dabei liegt die Schweiz mit einem Jahresverbrauch von etwa $64 \mathrm{~kg}$ Weißzucker pro Kopf weit vor allen anderen Staaten oder Erdteilen. Er ist knapp doppelt so hoch wie der Weißzuckerverbrauch pro Kopf in Europa, etwa vierfach so hoch wie der in Afrika oder Asien und

\footnotetext{
${ }^{1}$ http://www.zuckerverbaende.de.
} 
(in 1.000 t Rohwert)

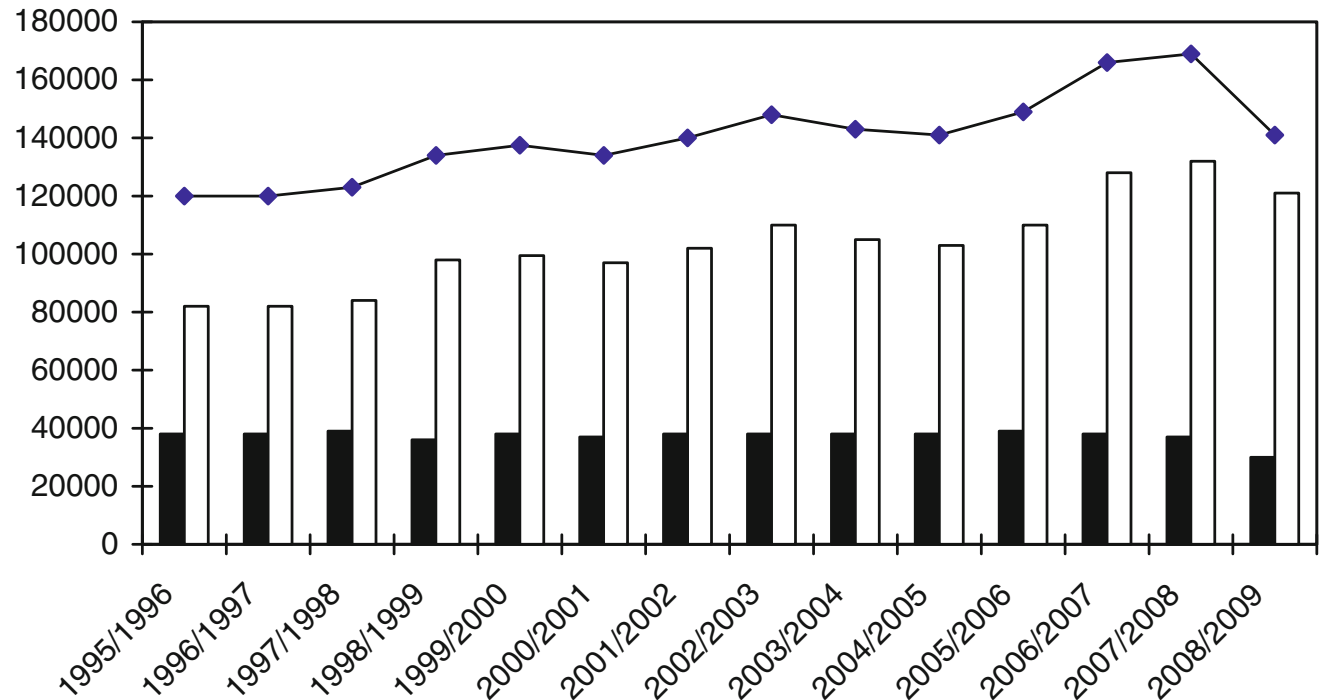

Abb. 1 Entwicklung der Weltzuckererzeugung insgesamt (schwarze Rhomben) im Zeitraum von 1995 bis 2009 im Vergleich zur Rohr- (weiße Balken) und Rübenzuckererzeugung (schwarze Balken) (nach Angaben der Wirtschaftlichen Vereinigung Zucker; http://www.zuckerverbaende.de) etwa 1,5fach so hoch wie der weltweite Weißzuckerbrauch pro Kopf. Zieht man außerdem den relativ hohen jährlichen Weißzuckerverbrauch von etwa $57 \mathrm{~kg}$ pro Kopf in Australien oder den von etwa $46 \mathrm{~kg}$ pro Kopf in Ozeanien in Betracht, so wird das Verlangen nach dem Süßen sicher durch die „eingeübten“ Ernährungsgewohnheiten unterstützt, sofern es durch die wirtschaftliche Lage des Einzelnen denn realisierbar ist.

Eine wirklich deutlich abnehmende Tendenz im Weißzuckerverbrauch pro Kopf ist während der vergangenen fünf Jahre nicht erkennbar (Abb. 2), wenn auch z. B. die Weltgesundheitsorganisation (WHO) im Jahr 2004 mit der „Global Strategy on Diet, Physical Activity and Health“ in den Richtlinien zur Bekämpfung ernährungsbedingter Krankheiten u. a. auch den verminderten Konsum von Zucker angemahnt hat. Wie schwer muss sich der Verbraucher auch tun, wenn er - wohl wissend um den gesundheitsabträglichen Einfluss eines zu hohen Zuckerkonsums - z. B. mit einem Satz wie „Die schwere Süße des Zuckers und der Sahne vermischt sich mit der herben Schärfe der Schokolade ${ }^{\alpha 2}$ zumindest gedanklich ins genüssliche Schwelgen versetzt werden kann.

Dessen ungeachtet scheint sich bei den Verbrauchern dennoch langsam die Einsicht durchzusetzen,

\footnotetext{
${ }^{2}$ Mayer V (1009) Mit kalter Hand gerollt. Die ZEIT, 10.12.2009, Nr. 51, S. 63.
}

dass angesichts Übergewicht und bewegungsarmen Lebenswandels eine sinnvoll gewählte, kalorienreduzierte Ernährung im Einzelfall durchaus gesundheitlich angebracht sein kann. Diese Tendenz drückt sich z. B. auch in dem vermehrten Bevorzugen von Erfrischungsgetränken aus, deren Zuckergehalt abgesenkt oder gar durch andere Süßstoffe ersetzt worden ist. Im Fall des Ersatzes durch synthetische Süßstoffe kommt es bei etlichen Verbrauchern allerdings zum Entscheidungsdilemma zwischen kalorienarmen oder natürlich gesüßten Erfrischungsgetränken.

Diese Entwicklung und dieser möglicherweise grundlegende Wandel im Verbraucherverhalten in Europa und Nordamerika ist bereits von der Lebensmittelindustrie erkannt und mögliche neue Verkaufsstrategien entwickelt worden. Es kann durchaus davon ausgegangen werden, dass einige davon schon in den nächsten vier oder fünf Jahren realisiert werden können.

Dies ist aus wirtschaftlicher Sicht umso prekärer, als gerade in diesem Zeitrahmen z. B. auf die deutschen Zuckerrübenanbauer noch nicht absehbare marktwirtschaftliche Veränderungen warten. Zum Beispiel ist nach Auskunft des Rübenanbauer- und Aktionärsverbands Nord, der gemeinsam mit sieben anderen Zuckerrübenanbauerverbänden im Dachverband Norddeutscher Zuckerrübenanbauer (DZN) die Interessen der Rübenanbauer gegenüber den Zuckerunternehmen und gegenüber deutschen und 


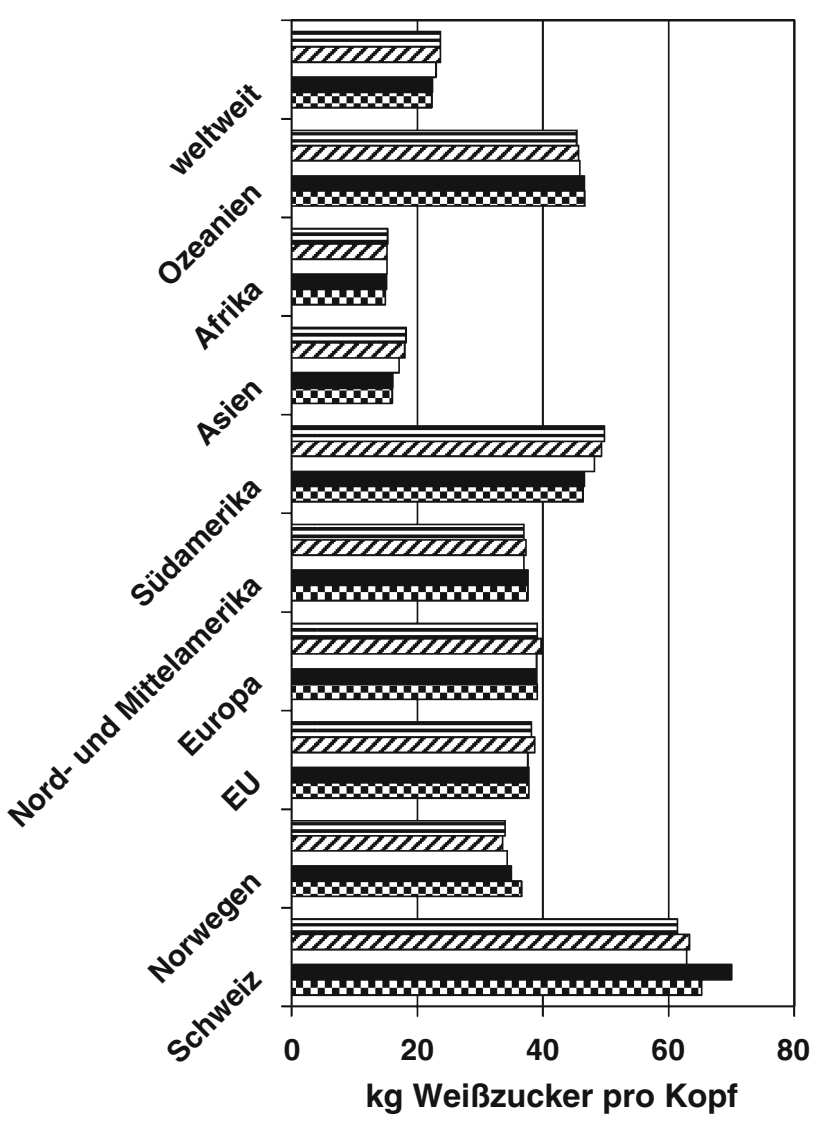

Abb. 2 Zuckerverbrauch in einigen Ländern sowie Erdteilen im Zeitraum 2004 bis 2009 (schwarz/weiße Quadrate = 2004/2005; schwarz = 2005/2006; weiß =2006/2007; diagonal schwarz gestreift $=2007 / 2008$; längs schwarz gestreift $=2008 / 2009$ ) (in kg Weißzucker pro Kopf) (nach Angaben der Wirtschaftlichen Vereinigung Zucker; http://www.zuckerverbaende.de)

europäischen politischen Institutionen vertritt, trotz der überdurchschnittlichen Ernte im Jahr 2009 von stabilen Preisen auszugehen. Bis zur Kampagne im Jahr 2014 gebe es einen bei 33,50€ pro Tonne fixierten Preis. Doch wie die Zuckermarktordnung der EU nach dem Jahr 2015 aussehen werde, sei äußerst fraglich. Bei einem steigenden Zuckerverbrauch weltweit seien die Exportmöglichkeiten politisch stark eingeschränkt worden. ${ }^{3}$

Bei dieser derzeitigen Ausgangslage, in der das gewohnte Süßungsmittel Zucker an Zuspruch durch die Verbraucher zu verlieren scheint, den synthetisch erzeugten Süßungsmitteln als nicht natürliche Stoffe nur geminderte Akzeptanz durch die Verbraucher entgegengebracht wird und außerdem noch eine wirtschaftspolitische Neuordnung des weltweiten Zuckermarktes bevorzustehen scheint, ist es aus Sicht der Lebensmittelindustrie nur allzu verständlich, dass

\footnotetext{
3 Köhler-Götze J (2009) Zufriedene Rübenbauern. Isenhagener Kreisblatt vom 9. Dezember 2009, S. 6.
}

Erfolg versprechende Alternativen zum Süßungsmittel Zucker aufgegriffen und in Hinblick auf zukünftigen Einsatz und Vermarktung weiter entwickelt werden. Als Beispiel dafür seien hier in Kürze die Ergebnisse des sehr instruktiven Vortrags von Dr. Ley (Fa. Symrise) auf der Fachtagung „Stevia“ - Ante Portas! am 22. Oktober 2009 in Hohenheim vorgestellt über die geschmackliche Beurteilung von sieben ausgewählten Steviolglykosid-Proben. Als Bewertungskriterien wurden ausgewählt: Adstringierend, bitter, lakritzartig, karamelartig, krautartig, sauer und vollmundig. Des Weiteren sollte das Süßprofil bestimmt werden, ob sich die Süße langsam entwickelt und wie schnell die Süßwahrnehmung wieder abnimmt. Als allgemeiner Eindruck wurde gefragt, ob das jeweils getestete Steviolglykosid eher unangenehm oder eher angenehm empfunden wird, sowie ob der geschmackliche Eindruck eher künstlich oder natürlich ist.

Aufgrund der vorläufigen Auswertung von 78 Bewertungsbogen kam Dr. Ley zu dem vorläufigen Befund, dass die geschmacklichen Eigenschaften der Stevia-Süßstoffe durch drei Faktoren beeinflusst werden: (a) die chemische Zusammensetzung der jeweiligen Stevia-Varietät, (b) die Anbauregion der jeweiligen Stevia-Varietät und (c) das Herstellungsverfahren der Stevia-Süßstoffe selbst. Zusammengenommen ergibt sich aus diesen Angaben gleichsam ein „,sensorischer Fingerprint“, der jedes Steviolglykosid hinreichend charakterisiert.

Soweit es bei der derzeit noch begrenzten Datenbasis nur mit Einschränkung erlaubt ist, jetzt schon belastbare Rückschlüsse aus den Ergebnissen dieser geschmacklichen Beurteilung zu ziehen, so gibt es aber nach den Untersuchungen von Dr. Ley Anzeichen dafür, dass Steviolglykoside mit einem deutlichen Rebaudiosid A-Anteil vorwiegend mit „sehr unangenehm“ bewertet wurden. Andererseits wurden Steviolglykosiden mit hoher Reinheit nicht unbedingt die beste Präferenz im Hinblick auf das Bewertungskriterium „Natürlichkeit“ verliehen.

Insgesamt scheint dem „Süßstoff-Sektor“ der Lebensmittelindustrie in den nächsten Jahren eine spannende Entscheidung bevorzustehen, welchen Weg sie aufgrund eines Spektrums durchaus widersetzlicher Interessen und Vorlieben einschlagen soll. Eine erste Einstimmung zu dieser Thematik können die hier vorgelegten Beiträge der Fachtagung „Stevia“ - Ante Portas! liefern. 